\title{
Estimating Long-Term Survival of Adults with Philadelphia Chromosome-Negative Relapsed/ Refractory B-Precursor Acute Lymphoblastic Leukemia Treated with Blinatumomab Using Historical Data
}

Arie Barlev • Vincent W. Lin · Aaron Katz $\cdot$ Kuolung Hu •

Ze Cong $\cdot$ Beth Barber

Received: June 27, 2016 / Published online: November 21, 2016

(c) The Author(s) 2016. This article is published with open access at Springerlink.com

\begin{abstract}
Introduction: Blinatumomab is a bispecific $\mathrm{T}$ cell-engaging antibody construct indicated for adult patients with relapsed/refractory (R/R) $\mathrm{Ph}(-) \quad$ B-precursor acute lymphoblastic leukemia (ALL), an aggressive disease with poor prognosis. A phase 2 single-arm clinical study showed that $43 \%$ of patients achieved CR/ CRh within two cycles and approximately 20\% of patients receiving blinatumomab were still alive after 2 years.
\end{abstract}

Methods: The objective of the current analysis was to estimate long-term survival of patients receiving blinatumomab beyond the observed time period in the clinical study using a large

Enhanced content To view enhanced content for this article go to http://www.medengine.com/Redeem/ 6227F0606C3830D5.

A. Barlev · V. W. Lin · Z. Cong $(\bowtie) \cdot$ B. Barber Global Health Economics, Amgen Inc.,

Thousand Oaks, CA, USA

e-mail: zcong@amgen.com

A. Katz

Center for Observational Research, Amgen Inc.,

Thousand Oaks, CA, USA

K. $\mathrm{Hu}$

Global Biostatistical Science, Amgen Inc.,

Thousand Oaks, CA, USA historical observational dataset. Conditional survival probabilities of blinatumomab-treated patients beyond month 60 were assumed to be the same as the US general population.

Results: At month 60, the estimated proportion of blinatumomab-treated patients alive was more than double that of historical patients $(12.6 \%$ vs $5.4 \%)$. The mean overall survival was 76.1 months for blinatumomab patients and 39.8 months for historical patients. Sensitivity analyses including additional follow-up data from the clinical study showed consistent results.

Conclusions: These findings suggest that blinatumomab provides substantial overall survival benefit to patients with (R/R) $\mathrm{Ph}(-)$ B-precursor ALL compared with salvage chemotherapy.

Funding: Amgen.

Trial Registration: ClinicalTrials.gov identifier NCT01466179 and NCT02003612.

Keywords: Acute lymphoblastic leukemia; Blinatumomab; Hematology; Long-term survival; Oncology 


\section{INTRODUCTION}

The prognosis for adult patients with Philadelphia chromosome-negative $(\mathrm{Ph}[-])$ B-precursor ALL who are refractory to treatment or experience relapse (R/R) is poor: over $90 \%$ die from the disease and their survival time is short (median OS is 3-5 months) [1-4]. These patients tend to be particularly young, with a median age of 34-39 years, and die, on average, 30 years prematurely [5-7]. In general, response to salvage treatment followed by hematopoietic stem cell transplant (HSCT) offers the only potential for cure and long-term survival $[1,4]$. Until recently, salvage treatment for adult patients with $\mathrm{R} / \mathrm{R}$ $\mathrm{Ph}(-)$ B-precursor ALL was limited to highly toxic multi-agent chemotherapy regimens. Most patients who receive these regimens endure repeated and prolonged hospitalizations due to the severity of the disease and the aggressiveness of the treatment itself [8-11].

Blinatumomab is a novel bispecific $\mathrm{T}$ cell engager $\left(\mathrm{BiTE}^{\circledR}\right)$ antibody construct that simultaneously binds CD3-positive cytotoxic $\mathrm{T}$ cells and CD19-positive B cells. Blinatumomab was approved for adults with $\mathrm{R} / \mathrm{R} \mathrm{Ph}(-)$ B-cell precursor ALL by the FDA in December 2014 and subsequently by the European Medicines Agency (EMA) in November 2015 [12, 13]. Regulatory approval of blinatumomab in the US was based on results from a single-arm phase 2 study in 189 adults with $\mathrm{R} / \mathrm{R} \mathrm{Ph}(-)$ B-precursor ALL [14]. Historical observational data from 1139 patients with R/R $\mathrm{Ph}(-)$ B-precursor ALL who received standard of care therapy in Europe and the US provided context for the interpretation of the single-arm clinical study [15]. The single-arm clinical study and the historical observational data have been compared for rates of complete remission (42.9\% vs $24 \%$ ) and overall survival (OS) at 1 (32.0\% vs $15.5 \%)$ and 3 years (13.8\% vs $6.2 \%)$
$[14,15]$. To date, the blinatumomab clinical study includes 3 years of survival follow-up data, after which time $13.8 \%$ of patients remained alive [Amgen data on file]. In comparison, $6.4 \%$ patients in the historical data were alive at 3 years. The historical observational dataset included up to 21 years of follow up, after which time between $2 \%$ and $3 \%$ of patients remained alive. Given the very small percentage of patients in the historical observational dataset who lived for at least 21 years, the proportion of patients receiving blinatumomab who were still alive after 3 years is of particular interest.

To estimate the effect of a treatment on long-term survival, the mean OS is a preferred endpoint to median OS because it captures the entire survival curve over the lifetime of a population [16]. The objective of this analysis was to estimate the long-term survival of patients with $\mathrm{R} / \mathrm{R} \mathrm{Ph}(-)$ B-cell precursor ALL receiving blinatumomab, leveraging the long duration of follow-up data available in the historical observational dataset.

\section{METHODS}

\section{Data Sources}

\section{Blinatumomab Clinical Trial}

This phase 2, open-label, single-arm study was conducted at sites across Europe and the US and enrolled patients over the period 2010-2014 (ClinicalTrials.gov NCT01466179) [14]. Eligible patients $(N=189)$ included men and women $\geq 18$ years of age with $\mathrm{Ph}(-)$ B-cell precursor ALL, with one of the following characteristics:

- relapsed/refractory disease with first remission duration $\leq 12$ months in first salvage;

- relapsed/refractory disease after first salvage; or 
- relapsed/refractory disease within 12 months of allogeneic HSCT.

Patients with these characteristics are considered particularly difficult to treat. Overall survival was collected as a secondary endpoint.

All procedures followed were in accordance with the ethical standards of the responsible committee on human experimentation (institutional and national) and with the Helsinki Declaration of 1964, as revised in 2013. Informed consent was obtained from all patients for being included in the study.

\section{Historical Observational Dataset}

A retrospective analysis was performed by combining previously collected data from adult R/R ALL patients in national study groups and large treatment centers in Europe and the US. (clinicaltrials.gov NCT02003612). Patients were initially selected from study group/site databases if they had a diagnosis of R/R Ph(-) B-precursor ALL between 1990 and 2013 , were aged $\geq 15$ years at the time of initial ALL diagnosis of ALL, and had no previous treatment with blinatumomab. Patients were further selected from the historical dataset based on key eligibility criteria from the blinatumomab clinical study, including age $\geq 18$ years at relapse, ALL that relapsed within 12 months from initial diagnosis, or relapsed after alloHSCT, or refractory to initial or subsequent treatments, or in second or later relapse. Patients with a first remission duration of $>12$ months in first salvage were excluded, unless they had a second relapse or a relapse within 12 months of receiving alloHSCT.

In total 1139 patients met these criteria, of whom survival data was available from 1112 patients [15]. Two-thirds of patients (67\%) were diagnosed after the year 2000 .
To calculate overall survival estimates, six strata were created based on known prognostic factors in R/R ALL (age, prior HSCT, and line of salvage). Combined survival outcomes were obtained by weighting the stratum-specific survival estimates according to the proportion of patients in the blinatumomab trial in each stratum [15].

\section{Estimation of Long-Term Overall Survival of Patients Receiving Blinatumomab}

Estimation of long-term survival was comprised of three phases. In the first phase, survival from months 0-25 was estimated using observed Kaplan-Meier survival data from the primary analysis of the blinatumomab clinical trial [14, Amgen data on file]. In the second phase, survival from months 26-60 was estimated using the historical observational dataset. Specifically, from months 26-60, we assumed that there was no additional treatment effect from blinatumomab-i.e., the survivors in the blinatumomab clinical trial at months 26-60 had the same monthly conditional survival probabilities as those in the historical observational dataset who received salvage chemotherapy $[17,18]$. In the third phase, since patients who survive for more than 5 years are considered to be cured of the disease, survival from month 60 onwards was estimated by applying the age-specific annual conditional probability of survival from the life tables of the US population.

\section{Calculation of Mean OS}

The mean OS for patients receiving blinatumomab was estimated by calculating the area under the curve (AUC) for all three phases combined. The same AUC calculation 
was also applied to calculate the mean OS for patients in the historical observational dataset.

\section{Validation of Estimates Using 3 Years of Observed Data From The Clinical Study}

Additional OS data from the blinatumomab clinical trial after 36 months of follow-up was used to validate the long-term OS estimation [Amgen data on file]. The three-phase method described above was replicated using the blinatumomab clinical trial data to estimate survival from months 0-36, extrapolating survival from months 37-60 using the historical observational dataset, and extrapolating from month 60 onwards using conditional survival probabilities from the general US population.

\section{RESULTS}

Details of the patient populations from the clinical and historical studies have been described previously, and rates of CR and OS were compared between the blinatumomab patients and the historical patients using appropriate adjusted analyses [14, 15].

\section{Observed OS Data}

In both the blinatumomab clinical trial and the historical observational dataset, the observed OS probability decreased quickly in the first 10 months and plateaued afterwards (Fig. 1).

Comparing the observed blinatumomab clinical trial survival curve to the historical observational dataset survival curve, there is a clear separation between the curves from month zero to month 25 (Fig. 1). At month 25 , the survival probability among patients from the blinatumomab clinical trial was

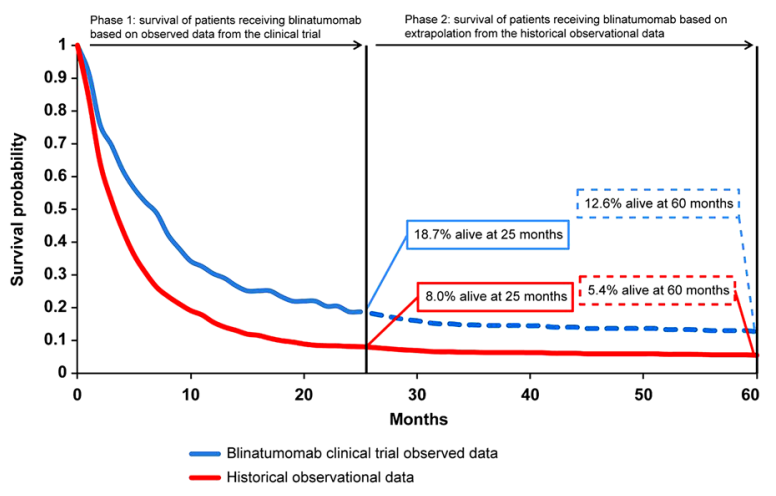

Fig. 1 5-year survival curves for patients receiving blinatumomab and historical dataset

$18.7 \%$, more than double the survival probability among patients from the historical observational dataset (8.0\%) based on the Kaplan-Meier estimate.

\section{OS Extrapolation up to 60 Months}

Based on extrapolation from the historical observational data, the OS probability at month 60 was $12.6 \%$ among patients from the blinatumomab clinical study and $5.4 \%$ in the historical observational dataset (Fig. 1).

\section{Mean OS}

The mean OS was calculated from the AUC of all three phases of the long-term survival estimation. The mean OS for patients in the blinatumomab clinical study was 76.1 months compared with 39.8 months for patients from the historical observational dataset (Table 1).

\section{Validation of Estimation}

The long-term OS estimation based on 36 months of OS data from the blinatumomab clinical trial was consistent with the estimation based on 25 months of trial data plus extrapolation with survival probabilities from 
Table 1 Mean overall survival estimation

\begin{tabular}{ll}
\hline & ${\text { Mean } \text { OS }^{\mathbf{a}} \text { (months) }}^{\text {Blinatumomab }}$ \\
Historical observational dataset & 36.06 \\
Difference & 36.22 \\
\hline
\end{tabular}

${ }^{a}$ Calculated by area under the curve (AUC) method

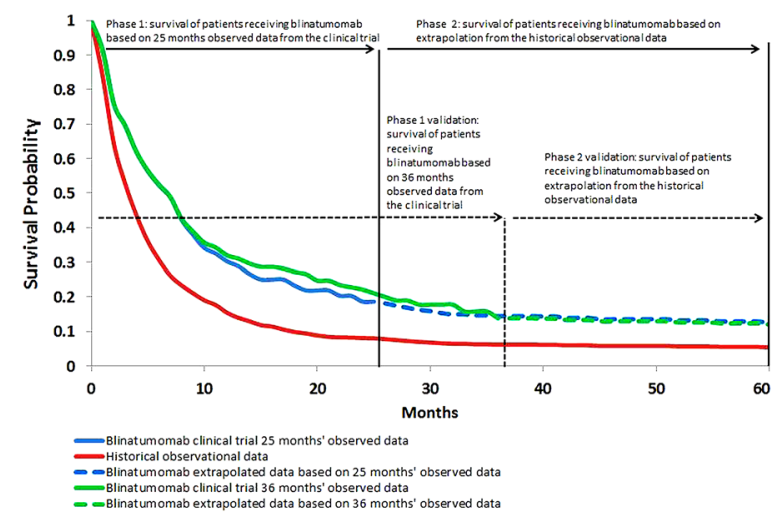

Fig. 2 Validation based on 36 months of observed data from the blinatumomab clinical trial

the historical observational dataset (Fig. 2). Using 36 months of survival data from the blinatumomab clinical trial, the survival probability at month 60 was $12.0 \%$ and the mean OS was 74.2 months.

\section{DISCUSSION}

This study estimated long-term survival for $\mathrm{R} / \mathrm{R}$ $\mathrm{Ph}(-)$ ALL patients receiving blinatumomab, using both the blinatumomab clinical trial results and long-term natural history data. Assuming no additional effects of blinatumomab beyond two years, the percentage of long-term survivors treated with blinatumomab is estimated to be more than double that of patients treated with salvage chemotherapy (12.6\% vs $5.4 \%$ after 5 years). The estimation technique was validated using an additional year of observed clinical trial data.
Similarly, the mean OS-a measure of overall survival over the lifetime of the populationwas also approximately doubled among blinatumomab-treated patients in comparison to patients in the historical observational dataset (76.1 vs 39.8 months).

The historical data set was pooled from European national study groups and large individual sites from Europe and the United States. Given rarity of $\mathrm{R} / \mathrm{R} \mathrm{Ph}(-)$ B-precursor ALL, the historical dataset represents the largest study of its kind in adults with this disease [13, 15], spanning over 20 years (1990-2013), During this time, no new effective treatments have emerged [3]. The increased use of paediatric-inspired protocols and improvements in supportive care and transplant realisation may have improved survival over time in some groups of adult $\mathrm{R} / \mathrm{R}$ ALL patients [19]. Two-thirds of patients in the historical dataset were diagnosed after the year 2000, the survival times were similar between the whole dataset and those patients diagnosed from 2000 onwards [15]. The historical data therefore appears to provide a valid reference for survival data extrapolation and comparison between novel agents and salvage chemotherapy.

It is worth noting that the assumed difference in survival between blinatumomab and salvage chemotherapy after the observed data time period in this study might be an underestimation. After the observed OS data, we assumed that the patients treated with blinatumomab had the same conditional survival probability as patients who received salvage chemotherapy. In fact, patients receiving blinatumomab can achieve a deep response, which is known to correlate with better long-term survival outcomes [20]. Among 81 patients with $\mathrm{R} / \mathrm{R} \mathrm{Ph}(-)$ B-precursor ALL who achieved complete remission $\left(\mathrm{CR} / \mathrm{CRh}^{*}\right), 82 \%$ 
also achieved minimal residual disease (MRD)-negativity within two cycles of blinatumomab [14]. In an earlier clinical study of 36 patients with $\mathrm{Ph}(-)$ B-cell precursor R/R ALL, 28\% of those treated with blinatumomab survived for at least 30 months, all of whom achieved an MRD-negative response [21]. These findings suggest that patients treated with blinatumomab might have higher conditional survival probability than patients receiving salvage chemotherapy. More patients in the blinatumomab clinical study proceed to receive transplants, possibly due to favourable CR rate, or higher rate of MRD negativity [14], which is highly correlated to positive HSCT outcome and better overall survival, compared to those who do not achieve MRD [22, 23].

With $12.6 \%$ of R/R ALL patients treated with blinatumomab expected to be alive after 5 years other treatment strategies are still required. Treatment with blinatumomab before relapse has been shown to produce high response rates and survival outcomes: $78 \%$ of patients with MRD-positive disease achieved a complete MRD response after 1 cycle of blinatumomab, and RFS was 58\% after 18 months [24]. In a smaller study in an MRD-positive population, after a median of 33 months follow up, $61 \%$ of blinatumomab-treated patients remained relapse-free [25]. These data raise the possibility that earlier intervention with blinatumomab may allow long-term remissions and reduce the need for salvage therapy.

The blinatumomab clinical trial enrolled $\mathrm{R} / \mathrm{R}$ $\mathrm{Ph}(-)$ B-precursor ALL patients who were selected for negative prognostic factors, which represents the majority of patients but nonetheless a subset of the total $\mathrm{R} / \mathrm{R} \mathrm{Ph}(-)$ B-precursor ALL population. Caution needs to be taken when extrapolating the results from this study to the broader patient group.
Limitations of this study are inherent in the use of historical data to compare and extrapolate survival outcomes with data from a clinical study. These include the assumption of no additional effects of blinatumomab beyond two years, potential changes in treatment practices over time in the clinical vs historical populations and differences in transplant realisation rates. Nonetheless, the superior survival outcomes with blinatumomab treatment over standard of care have recently been confirmed in a phase 3 study [26].

\section{CONCLUSION}

Compared to salvage chemotherapy, based on this analysis, blinatumomab provides substantial overall survival benefit to patients with R/R Ph(-) B-precursor ALL.

\section{ACKNOWLEDGEMENTS}

The study, article processing charges, and the open access charge were funded by Amgen. All named authors meet the International Committee of Medical Journal Editors (ICMJE) criteria for authorship for this manuscript, take responsibility for the integrity of the work as a whole, and have given final approval to the version to be published. All authors had full access to all of the data in this study and take complete responsibility for the integrity of the data and accuracy of the data analysis. Editorial assistance in the preparation of this manuscript was provided by James O'Kelly, an employee of Amgen.

Disclosures. Arie Barlev was an employee of Amgen at the time the study was conducted. Vincent Lin is an employee and shareholder in Amgen. Aaron Katz is an employee and 
shareholder in Amgen. Kuolung $\mathrm{Hu}$ is an employee and shareholder in Amgen. Ze Cong is an employee and shareholder in Amgen. Beth Barber is an employee of and shareholder in Amgen.

\section{Compliance with Ethics Guidelines. All} procedures followed in the blinatumomab clinical study were in accordance with the ethical standards of the responsible committee on human experimentation (institutional and national) and with the Helsinki Declaration of 1964, as revised in 2013. Informed consent was obtained from all patients for being included in the study. The historical study was based on data collected from previously-conducted studies.

Data Availability. The datasets during and/ or analyzed during the current study are available from the corresponding author on reasonable request.

Open Access. This article is distributed under the terms of the Creative Commons Attribution-NonCommercial 4.0 International License (http://creativecommons.org/licenses/ by-nc/4.0/), which permits any noncommercial use, distribution, and reproduction in any medium, provided you give appropriate credit to the original author(s) and the source, provide a link to the Creative Commons license, and indicate if changes were made.

\section{REFERENCES}

1. Fielding AK, Richards SM, Chopra R, et al. Outcome of 609 adults after relapse of acute lymphoblastic leukemia (ALL); an MRC UKALL12/ECOG 2993 study. Blood. 2007;109:944-50.

2. O'Brien S, Thomas D, Ravandi F, et al. Outcome of adults with acute lymphocytic leukemia after second salvage therapy. Cancer. 2008;113:3186-91.
3. Kantarjian HM, Thomas D, Ravandi F, et al. Defining the course and prognosis of adults with acute lymphocytic leukemia in first salvage after induction failure or short first remission duration. Cancer. 2010;116:5568-74.

4. Gokbuget N, Stanze D, Beck J, et al. Outcome of relapsed adult lymphoblastic leukemia depends on response to salvage chemotherapy, prognostic factors, and performance of stem cell transplantation. Blood. 2012;120:2032-41.

5. Kenderian SS, Al-Kali A, Gangat $\mathrm{N}$, et al. Monosomal karyotype in Philadelphia chromosome-negative acute lymphoblastic leukemia. Blood Cancer J. 2013;3:e122.

6. Maury S, Huguet F, Leguay $\mathrm{T}$, et al. Adverse prognostic significance of CD20 expression in adults with Philadelphia chromosome-negative B-cell precursor acute lymphoblastic leukemia. Haematologica. 2010;2:324-8.

7. Katz AJ, Chia VM, Schoonen WM, Kelsh MA. Acute lymphoblastic leukemia: an assessment of international incidence, survival, and disease burden. Cancer Causes Control. 2015;11:1627-42.

8. Barlev A, Lin VW, Song X. Burden of hospitalization in relapsed acute lymphoblastic leukemia. Curr Med Res Opin. 2016. [Epub ahead of print].

9. Dombret $\mathrm{H}$, Thomas $\mathrm{X}$, Chevallier $\mathrm{P}$, et al. Healthcare burden and economic cost of hospitalisation during chemotherapy for adult patients with Ph-negative B-precursor relapsed or refractory (R/R) acute lymphoblastic leukaemia (ALL) in France. Value Health. 2015;18:A443.

10. Kreuzer K, Stuhlmann R, Lebioda A, Reitan J, Barber B, Barlev A. Hospitalisations among adult patients with Ph-negative B-precursor relapsed or refractory (R/R) acute lymphoblastic leukaemia (ALL) receiving chemotherapy in Germany: a retrospective chart review. Value Health. 2015;18:A443.

11. Pagano L, Oberti M, Esposito B, Reitan J, Barlev A, Barber B, Ferrara F. Retrospective chart review of hospitalisations during chemotherapy for adult patients with Ph-negative B-precursor relapsed or refractory (R/R) acute lymphoblastic leukaemia (ALL) in Italy. Value Health. 2015;18:A443.

12. Blinatumomab USPI: http://pi.amgen.com/united states/blincyto/blincyto_pi_hcp_english.pdf. Accessed 5 May 2016.

13. Blinatumomab EPAR: http://www.ema.europa.eu/ docs/en_GB/document_library/EPAR_-_Public assessment_report/human/003731/WC500198227. pdf. Accessed 25 Feb 2016. 
14. Topp MS, Gokbuget N, Stein AS, et al. Safety and activity of blinatumomab for adult patients with relapsed or refractory B-precursor acute lymphoblastic leukaemia: a multicentre, single-arm, phase 2 study. Lancet Oncol. 2015;16:57-66.

15. Gökbuget N, Kelsh M, Chia V, et al. Blinatumomab versus historical standard therapy of adult relapsed/ refractory acute lymphoblastic leukaemia. Blood Cancer J Blood Cancer J. 2016;6(9):e473.

16. Latimer NR. Survival analysis for economic evaluations alongside clinical trials-extrapolation with patient-level data: inconsistencies, limitations, and a practical guide. Med Decis Making. 2013;33:743-54.

17. Demiris N, Sharples LD. Bayesian evidence synthesis to extrapolate survival estimates in cost-effectiveness studies. Stat Med. 2006;25:1960-75.

18. Drummond M, Sculpher MJ, Torrance GW, O'Brien BJ, Stoddart GL. Methods for the economic evaluation of health care programmes. 3rd ed. Oxford: Oxford University Press; 2005.

19. Pui $\mathrm{CH}$, Carroll WL, Meshinchi S, Arceci RJ. Biology, risk stratification, and therapy of pediatric acute leukemias: an update. J Clin Oncol. 2011;29:551-65.

20. Hoelzer D. Monitoring and managing minimal residual disease in acute lymphoblastic leukemia. Am Soc Clin Oncol Educ Book. 2013:290-93.

21. Zugmaier G, Gökbuget $\mathrm{N}$, Klinger $\mathrm{M}$, et al. Long-term survival and T-cell kinetics in relapsed/ refractory ALL patients who achieved MRD response after blinatumomab treatment. Blood. 2015;126:2578-84.

22. Jabbour E, Short NJ, Jorgensen JL, et al. Differential impact of minimal residual disease negativity according to the salvage status in patients with relapsed/refractory B-cell acute lymphoblastic leukemia. Cancer. 2016. [Epub ahead of print].

23. Ravandi F, Jorgensen JL, O'Brien SM, et al. Minimal residual disease assessed by multi-parameter flow cytometry is highly prognostic in adult patients with acute lymphoblastic leukaemia. Br J Haematol. 2016;172:392-400.

24. Gökbuget $\mathrm{N}$, Dombret $\mathrm{H}$, Bonifacio $\mathrm{M}$, et al. Long-term outcomes after blinatumomab treatment: follow-up of a phase 2 study in patients (pts) with minimal residual disease (MRD) positive B-cell precursor acute lymphoblastic leukemia (ALL). Blood. 2015;126:680.

25. Topp MS, Gökbuget $\mathrm{N}$, Zugmaier $\mathrm{G}$, et al. Long-term follow-up of hematologic relapse-free survival in a phase 2 study of blinatumomab in patients with MRD in B-lineage ALL. Blood. $2012 ; 120: 5185-7$

26. Topp MS, Stein A, Gökbuget N, Fielding AK, Schuh A, Ribera JM, et al. Blinatumomab improved overall survival in patients with relapsed or refractory Philadelphia negative B-cell precursor acute lymphoblastic leukemia in a randomized, open-label phase 3 study (TOWER). Haematologica. 2016;101(s1):S149. 